\title{
Mielolipoma retroperitoneal perirrenal: reporte de un caso
}

\section{Perirrenal retroperitoneal myelolipoma: a case report}

\author{
Martín Andrada*, Franco J. Signorini, Álvaro Alcaraz, Enzo A. Giordano y \\ Lucio R. Obeide \\ Servicio de Cirugía General, Hospital Privado Universitario de Córdoba, Córdoba, Argentina
}

\begin{abstract}
Resumen
El mielolipoma es un tumor benigno de baja incidencia cuya localización más frecuente son las glándulas suprarrenales. Histológicamente se caracteriza por células con precursores mieloides y eritroides mezcladas con tejido adiposo maduro. El diagnóstico en general es incidental en una prueba de imagen. Clínicamente cursa asintomático, aunque los de mayor tamaño tienen más riesgo de complicaciones como sangrado o efecto de masa. Los hallazgos incidentales $<4 \mathrm{~cm}$ se deben controlar con imágenes; los $>7 \mathrm{~cm}$, o que generen síntomas, deben ser tratados de forma quirúrgica. Se reporta un caso de mielolipoma extrasuprarrenal en un paciente de 78 años.
\end{abstract}

Palabras clave: Glándulas suprarrenales. Hallazgos incidentales. Mielolipoma.

\begin{abstract}
Myelolipoma is a relatively rare benign tumor which is most commonly located in the adrenal glands. Histologically is characterized by eritroid and myeloid precursor cells intermixed with mature adipose tissue. The diagnosis is generally incidental in abdominal imaging studies. Clinically most are asymptomatic, nevertheless larger tumors are at greater risk for complications such as hemorrhage or compression of surrounding structures. Incidental findings smaller than $4 \mathrm{~cm}$ should be followed-up by imaging. Tumors measuring more than $7 \mathrm{~cm}$ or those that are symptomatic a surgical approach is mandated. We present the case of a 78-year-old man with an extra-adrenal myelolipoma.
\end{abstract}

Key words: Adrenal glands. Incidental findings. Myelolipoma.

\section{Introducción}

El mielolipoma es un tumor benigno constituido por tejido adiposo maduro y componentes hematopoyéticos en su interior ${ }^{1}$. La localización más frecuente es la glándula suprarrenal, aunque puede localizarse en la región presacra, el retroperitoneo, la pelvis y el mediastino ${ }^{2}$. El principal desafío es diferenciarlo de tumores malignos de características similares, como el liposarcoma. La tomografía computarizada (TC) y la resonancia magnética (RM) son pruebas de imagen útiles. Cuando la localización es la glándula suprarrenal, el diagnóstico es certero, ya que es la única patología conocida compuesta por tejido adiposo en

\footnotetext{
Correspondencia:

*Martín Andrada

Obispo Oro, 479, 3B

Fecha de recepción: 03-04-2020

C.P. 5000, Córdoba capital, Córdoba, Argentina

E-mail: martin.andrada.d@gmail.com

0009-7411/@ 2020 Academia Mexicana de Cirugía. Publicado por Permanyer. Este es un artículo open access bajo la licencia CC BY-NC-ND (http://creativecommons.org/licenses/by-nc-nd/4.0/).
} 
dicho órgano, pero no así cuando es extrasuprarrenal, en cuyo caso se convierte en un verdadero desafío abordar el diagnóstico ${ }^{3}$. A continuación se presenta el caso de un paciente de sexo masculino con hallazgo incidental de mielolipoma retroperitoneal perirrenal en una TC solicitada para el control de una diverticulosis colónica.

\section{Caso clínico}

Varón de 78 años con antecedente de hipertensión arterial, diabetes mellitus tipo 2, hiperplasia prostática benigna y diverticulosis colónica, que es sometido a una TC de control (Figs. 1 -2) en la que se encuentra, a nivel retroperitoneal, infrarrenal derecho, una lesión redondeada, de contornos mal definidos, con densidad grasa y densidad de partes blandas, de $85 \mathrm{~mm}$ de diámetro. Se solicita RM con gadolinio (Figs. 3-4) que informa lesión de $85 \mathrm{~mm}$ de diámetro máximo que presenta componente con intensidad grasa extracelular y componente de partes blandas con contenido adiposo intracelular, sin realce tras la administración de contraste.

Se discute el caso en el comité de cirugía oncológica, al ser una lesión asintomática pero de características inciertas por imágenes, y se decide su resección quirúrgica.

Se realizó laparotomía mediana suprainfraumbilical y se inspeccionó la cavidad abdominal, constatando la ausencia de implantes peritoneales y hepáticos. Se procedió a realizar maniobra de Cattell y Kocher amplia, luego se disecó el uréter derecho y se liberó el tumor retroperitoneal por la cara anterior del músculo psoas. Se realizó disección y liberación del tumor de la cápsula renal derecha y se extrajo la pieza quirúrgica. Se colocó drenaje en el sitio de tumorectomía y finalizó el procedimiento con el cierre de la pared abdominal.

El paciente cursa el posoperatorio inmediato en la unidad de cuidados intensivos, por 3 días. Al quinto día, ya en sala común, presenta un cuadro de oclusión intestinal confirmado por TC que se interpreta como íleo mecánico, por lo que se decide realizar una nueva laparotomía, en la que se observa oclusión del intestino delgado secundaria a bridas. El paciente evoluciona favorablemente y se otorga el alta hospitalaria a los 8 días de la segunda cirugía.

En el estudio de anatomía patológica, las secciones histológicas mostraron adipocitos maduros mezclados con islas de fibrosis y elementos hematopoyéticos compuestos de células mieloides y eritroides, linfocitos, células plasmáticas y megacariocitos raros. La inmunohistoquímica informó de algunas células proliferantes positivas para S100 (Figs. 5-6).

El resultado de la hibridación fluorescente in situ (FISH) fue negativo para amplificación del gen MDM2 (mouse doublé minute 2) en $12 q 15$.

\section{Discusión}

El mielolipoma se caracteriza por ser una lesión bien circunscrita que contiene varias combinaciones de precursores mieloides y eritroides mezclados con tejido adiposo maduro.

La localización más frecuente es la glándula suprarrenal, pero también puede hallarse en el retroperitoneo presacro, el retroperitoneo perirrenal, el mediastino, el hígado, el estómago y la fascia muscular. Es un tumor mesenquimatoso benigno, poco común, con una incidencia del $0.02-0.4 \%$, más frecuente en las mujeres que en los hombres, la mayoría unilaterales y su tamaño es variable. La localización en regiones extrasuprarrenales es más común en los pacientes de mayor edad ${ }^{4}$. En la actualidad solo hay reportados nueve casos de localización extraadrenal perirrenal ${ }^{5}$.

La etiología es controversial. Se cree que pueden originarse a partir de la reactivación de focos peritoneales primitivos de hematopoyesis extramedular bajo estrés patológico, como anemia grave, sepsis o enfermedad mieloproliferativa. Otra teoría es la metaplasia de células mesenquimatosas suprarrenales corticales no comprometidas o células madre hematopoyéticas que normalmente migran a la glándula suprarrenal durante el desarrollo intrauterino ${ }^{6}$.

La amplificación del gen MDM2 se ha observado hasta en el 99\% de los liposarcomas bien diferenciados y hasta en el $30 \%$ de otros tipos de sarcomas. Esta prueba ayuda a distinguir entre tumor lipomatoso atípico, liposarcoma bien diferenciado y algunas lesiones benignas o no neoplásicas, como inflamación en una lesión adipocítica benigna o angiofibroma celular. La región cromosómica 12q13-q15 a menudo se ve afectada por translocaciones y amplificaciones en el sarcoma de tejidos blandos en humanos. Esta región incluye el gen MDM2 que inhibe la actividad transcripcional de p53 uniéndose a este y moviendo la proteína al citoplasma. Esto da como resultado la inactivación del supresor tumoral y la formación de tumores que finalmente conduce al cáncer. Se ha documentado el uso de MDM2 como diagnóstico diferencial de ayuda para el sarcoma. 


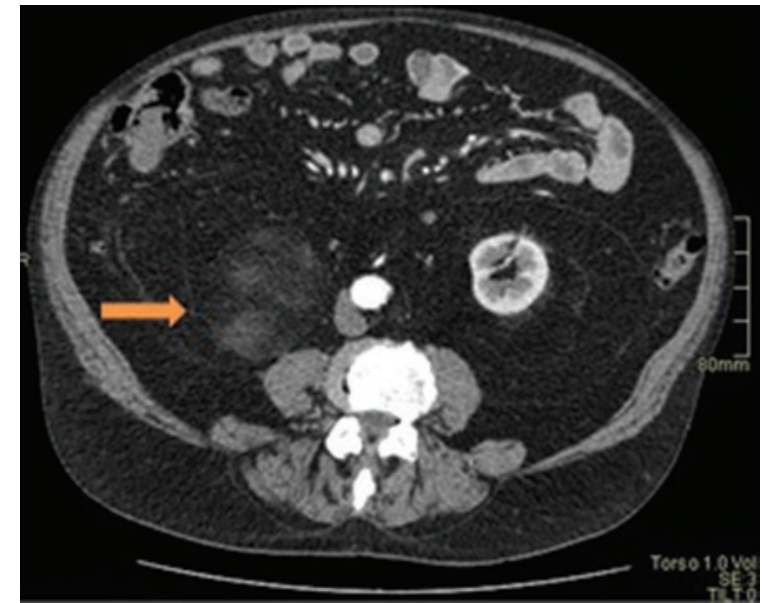

Figura 1. Tomografía computarizada, corte axial, que muestra una lesión blastomatosa de $85 \mathrm{~cm}$ de diámetro.

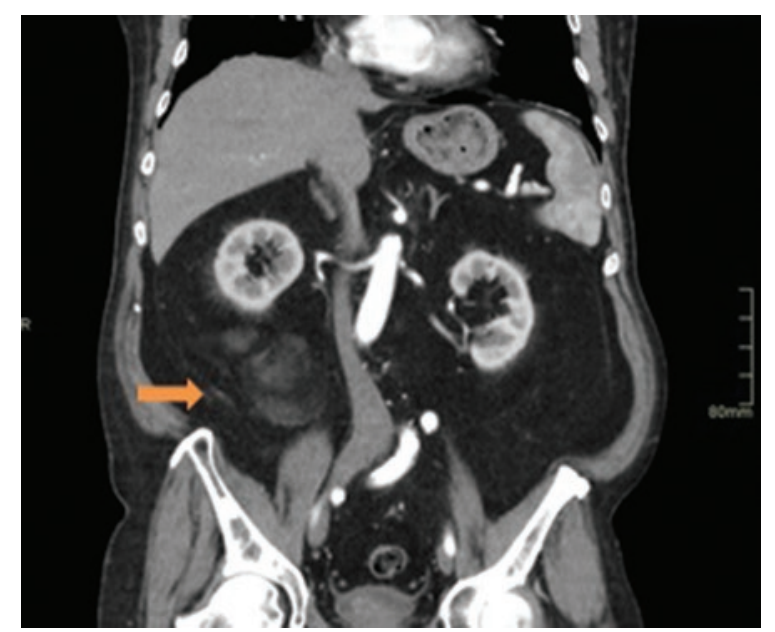

Figura 2. Tomografía computarizada, corte coronal, que muestra una lesión redondeada infrarrenal derecha.

La presentación clínica varía de acuerdo con el tamaño y la localización de la lesión. Sus síntomas principales se deben al efecto de masa (dolor de espalda o abdominal, hipertensión arterial, hematuria o dolor en el sitio del tumor), pero a pesar de esto la mayoría son incidentalomas?.

La hemorragia aguda es la complicación más frecuente y puede manifestarse como dolor, náuseas, vómitos, hipotensión y anemia. Esta complicación ocurre en las lesiones más grandes $(>10 \mathrm{~cm}$ de diámetro) y es más frecuente en los hombres.

Como método complementario para el diagnóstico de este tipo de lesión, la TC y la RM son de utilidad; sin embargo, una masa grasa en el retroperitoneo representa un desafío para los médicos, ya que el

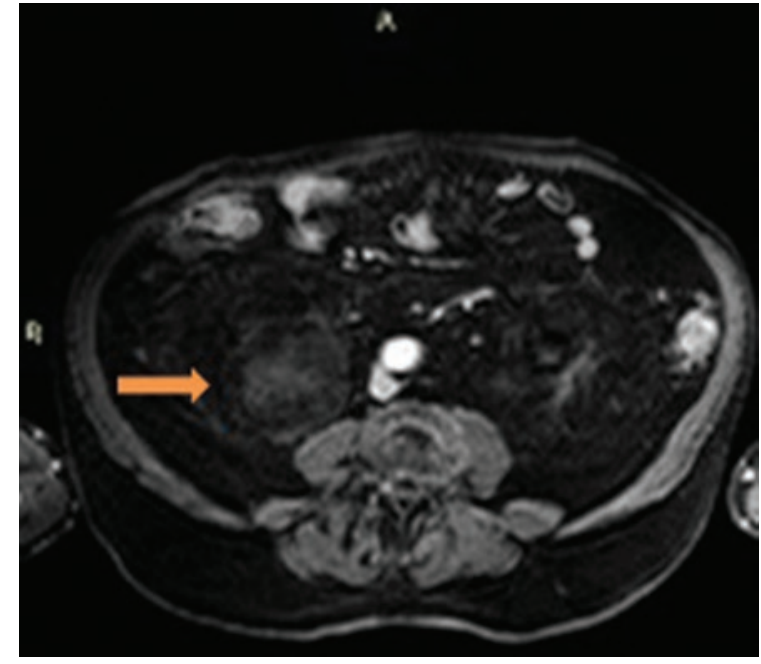

Figura 3. Resonancia magnética de abdomen, corte axial, que muestra una lesión con contenido adiposo intracelular, sin realce con contraste intravenoso.

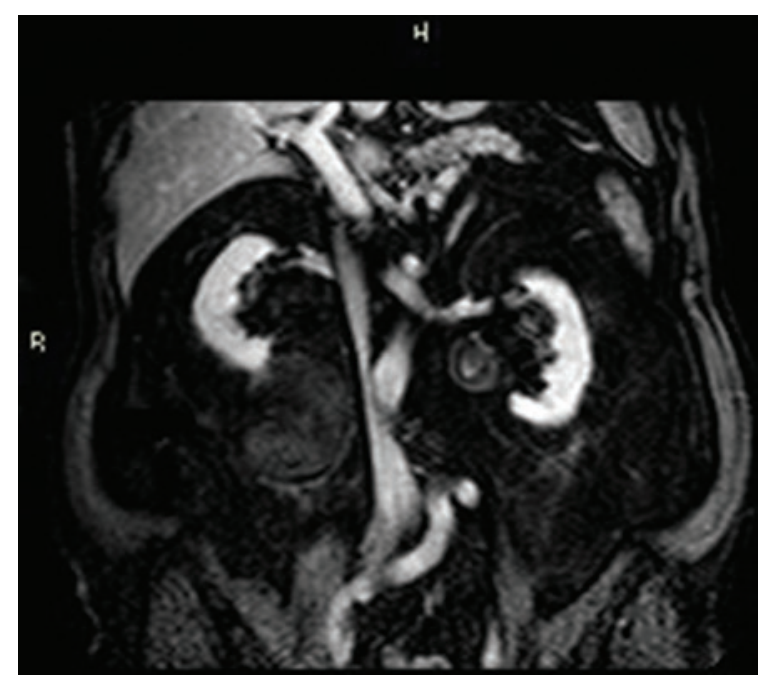

Figura 4. Resonancia magnética de abdomen, corte coronal, que muestra una lesión hipointensa infrarrenal derecha.

diagnóstico diferencial incluye angiomiolipoma, teratoma retroperitoneal y liposarcoma bien diferenciado.

Es controversial la realización de biopsia para el diagnóstico definitivo de la lesión. Muchos cirujanos consideran que la biopsia es una contraindicación en cualquier lesión que sea resecable, debido a los riesgos de siembra en el tracto de la aguja de biopsia con células potencialmente malignas, o de infectar la lesión. Otros sugieren que se debe considerar una biopsia preoperatoria, ya que los resultados patológicos intervienen en el manejo, particularmente para las lesiones que son grandes o que invaden las estructuras adyacentes ${ }^{8}$. 


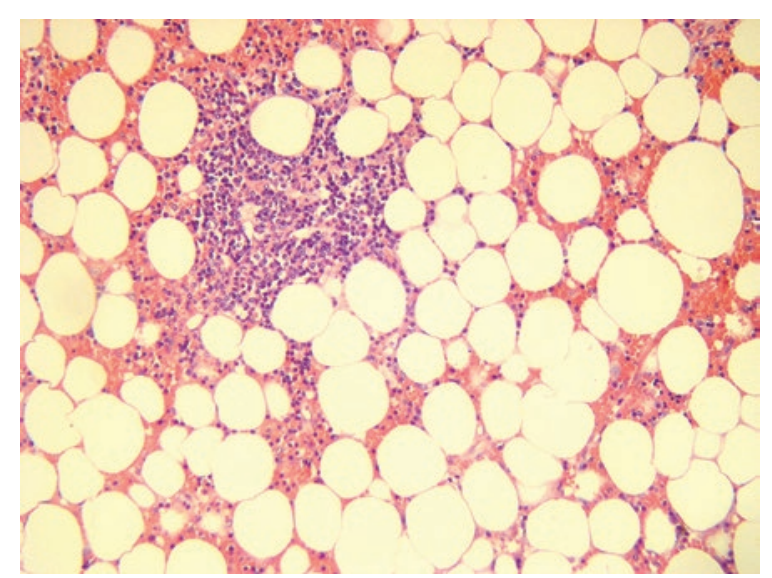

Figura 5. Anatomía patológica: tinción de hematoxilina-eosina.

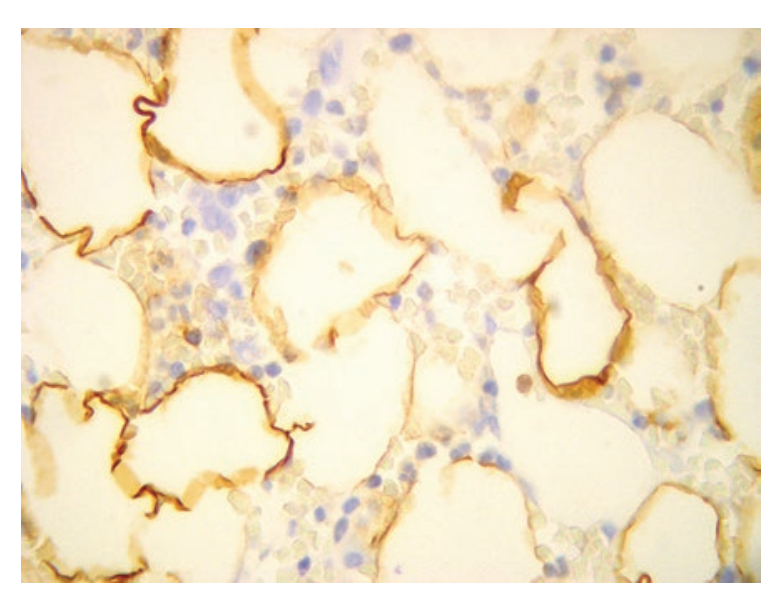

Figura 6. Anatomía patológica: inmunohistoquímica positiva para S100.

No existe un tratamiento estándar para este tipo de tumores. Se recomienda que los tumores pequeños y asintomáticos $(<4 \mathrm{~cm})$ se monitoricen con pruebas de imagen, mientras que los tumores sintomáticos grandes $(>7 \mathrm{~cm})$ se deben extirpar quirúrgicamente ${ }^{4}$.

La vía de abordaje videolaparoscópica es el método de referencia para tratar lesiones adrenales benignas como el mielolipoma suprarrenal ${ }^{9}$. A pesar de esto, solo hay reportado un caso en la literatura de abordaje videolaparoscópico para el tratamiento del mielolipoma de localización extraadrenal perirrenal. En este caso reportado se decidió utilizar la vía laparoscópica ya que previamente se realizó una biopsia percutánea que informaba de una lesión benigna ${ }^{10}$. En nuestro caso, el objetivo fue una cirugía curativa desde el punto de vista oncológico, por lo que se decidió realizar un abordaje convencional ya que la lesión presentaba una naturaleza incierta en las imágenes. Ante la sospecha de malignidad, muchos autores no recomiendan la realización de biopsias previas.

\section{Conflicto de intereses}

Los autores declaran que el reporte de este caso se llevó a cabo en ausencia de cualquier relación comercial o financiera que pudiera ser interpretada como un posible conflicto de intereses.

\section{Responsabilidades éticas}

Protección de personas y animales. Los autores declaran que los procedimientos seguidos se conformaron a las normas éticas del comité de experimentación humana responsable y de acuerdo con la Asociación Médica Mundial y la Declaración de Helsinki.

Confidencialidad de los datos. Los autores declaran que han seguido los protocolos de su centro de trabajo sobre la publicación de datos de pacientes.

Derecho a la privacidad y consentimiento informado. Los autores han obtenido el consentimiento informado de los pacientes y/o sujetos referidos en el artículo. Este documento obra en poder del autor de correspondencia.

\section{Bibliografía}

1. Spanra R, Saleh HA, Khatib G. Fine needle aspiration diagnosis of extra adrenal myelolipoma presenting as a pleural mass. A case report. Acta Cytol. 1999;43:295-8.

2. Doddi S, Singhal T, Leake T, Sinha P. Management of an incidentally found large adrenal myelolipoma: a case report. Cases J. 2009;2:8414.

3. Dann PH, Krinsky GA, Israel GM. Case 135: presacral myelolipoma. Radiology. 2008;248:314-6

4. Kammen BF, Elder DE, Fraker DL, Siegelman ES. Extraadrenal myelolipoma: MR imaging findings. AJR Am J Roentgenol. 1998;171:721-3.

5. Hajiran A, Morley C, Jansen R, Kandzari S, Bacaj P, Zaslau S, et al. Perirenal extra-adrenal myelolipoma. World J Clin Cases. 2014;2:279-83.

6. Amin MB, Tickoo SK, Schultz D. Myelolipoma of the renal sinus. An unusual site for a rare extra-adrenal lesion. Arch Pathol Lab Med. 1999;123:631-4.

7. Daneshmand S, Quek ML. Adrenal myelolipoma: diagnosis and management. Urol J. 2006;3:71-4.

8. Suárez-Peñaranda JM, Bermúdez Naveira A, Fraga M, Aliste-Santos C, Cordeiro C, Muñoz-Barús JI. Unusual forms of adrenal and extra-adrenal myelolipomas. Int J Surg Pathol. 2014;22:473-7.

9. Castillo O, Sánchez-Salas R, Vidal I. Laparoscopic adrenalectomy. Minerva Urol Nefrol. 2008;60:177-84.

10. Beiko D, Roldan H, Sengupta SK, George RL. Laparoscopic excision of a large extra-adrenal perirenal myelolipoma. Can Urol Assoc J. 2010;4:E39-41. 